DOI: https://doi.org/10.11144/Javeriana.upsy19.csps

\title{
La Coalición S y el Plan S: Implicaciones para los ecosistemas de conocimiento en América Latina
}

\author{
WILSON LÓPEZ-LÓPEZ \\ Pontificia Universidad Javeriana, Colombia \\ ORCID: http://orcid.org/0000-0002-2964-0402
}

Para citar esta editorial: López-López, W. (2019). La Coalición S y el Plan S: Implicaciones para los ecosistemas de conocimiento en América Latina. Universitas Psychologica, 19. https://doi.org/10. 11144/Javeriana.upsy19.csps
El año pasado fui nombrado embajador el Plan S, creo que esta tarea se encuentra alineado con mi recorrido comprometido con diversas iniciativas de promover el acceso abierto. Una buena parte de la comunidad no tiene información de la iniciativa y de sus implicaciones para los ecosistemas de producción y comunicación científica en la región, por lo que me he propuesto realizar esta editorial.

El Plan S es una iniciativa que, en palabras de Schiltz (2018), presidente de Science Europe, surge dentro del marco de las iniciativas de Berlín 2003 y en la declaración de 2016 en la que todos los ministros de ciencia de la Unión Europea (UE), reunidos en el Consejo de Competitividad, se comprometieron para que todas las publicaciones científicas europeas sean accesibles para el año 2020.

Resulta claro que los investigadores pueden publicar donde quieran, al igual que es evidente que los muros de pago de las revistas impiden que las comunidades puedan acceder a los contenidos, lo cual genera una ruptura ética; la gente que paga impuestos, que en parte son destinados a la financiación de la ciencia, tiene derecho a conocer los resultados de esa inversión. Impedir el acceso libre a información financiada con recursos públicos contribuiría a profundizar las brechas de desigualdad. Si los estados, a través de las instituciones responsables de la ciencia y la educación promueven o toleran estos muros de pago están contribuyendo a la desigualdad, lo cual implica un costo político pues afecta directamente los principios de las democracias liberales, entre los que se incluye el garantizar el acceso a la información, tal como lo ha señalado la UNESCO en sus diversas declaraciones (UNESCO, 2011).

En contraste, la manera en la que los principios del Plan S están formulados evidencia como los sistemas de evaluación e incentivos están completamente controlados por 
los actores comerciales que manejan la industria editorial de conocimiento. Así, empresas como Elsevier no sólo controlan miles de revistas de pago, sino también es dueña de sistemas como Scopus, que es usado como criterio de evaluación para la asignación de incentivos en buena parte de América Latina. Clarivate es dueña de Web of Science (WoS) y su sistema de evaluación Journal of Citations Report (JCR), que también es utilizado como recurso de evaluación. Estas dos compañías afirman que no son responsables de que las instituciones usen su información cienciométricas; ambas han escrito sobre las limitaciones de los indicadores que producen e incluso han evidenciado que no es adecuado el uso de estos como instrumentos de evaluación y generación de incentivos (FlórezCarranza, 2018; López-López, 2019a). No obstante, deberíamos cuestionar la transparencia sobre la forma de compra de estos sistemas de información tanto en agencias gubernamentales como en universidades, asegurándonos de que los procedimientos en los que se basan sean transparentes y justos. Al igual que debemos examinar críticamente los procesos de discusión y toma de decisiones que guían a la adopción de criterios de evaluación de la producción por parte de los tomadores de decisiones en los sistemas gubernamentales e institucionales de evaluación, asignación de incentivos y acreditación.

Es claro que muchos decisores no tienen conocimiento apropiado y perspectiva del funcionamiento de los ecosistemas de conocimiento. Los intereses y tensiones entre los actores, los procesos y las apropiaciones producen distorsiones que afectan la gobernanza y con frecuencia tienen implicaciones éticas en el manejo de los ecosistemas de conocimiento (López-López, 2019b).

Por esta razón, el Plan $S$ asume la declaración DORA (2012) cómo uno de sus 10 principios. Esta declaración promueve procesos de evaluación no centrados en los indicadores globales y sesgados de la revista, proponiendo al artículo como unidad de conocimiento que debe ser el núcleo central de la evaluación. En cambio, DORA aboga el uso de una variedad de criterios de evaluación basados en la calidad.
El Plan S es taxativo en estar contra las revistas hibridas (revistas por suscripción con algunos artículos en acceso abierto). Al mismo tiempo, entiende que existen empresas editoriales que tienen unas estructuras de costos y por tanto reconoce "acuerdos transformacionales" en los que las tarifas por suscripción se compensan con las de "publicación y lectura". La Coalición S ve estos como acuerdos de transformación como parte de un proceso de transición breve al acceso abierto completo (cOAlition S, 2019).

En América Latina esto tiene dos caras. Por un lado, la mayor parte de nuestras revistas son completamente de acceso abierto y la región es pionera en una larga tradición de repositorios que están integrados con este modelo de publicación. Primero, en la Universidad Nacional Autónoma de México iniciativas como Latindex, un directorio en acceso abierto, y las bases de datos Periódica y Clase desde finales de los años 70 se encontraron en esta dirección. En 1998, la declaración de San José llevó a la creación del Sistema Latinoamericano y del Caribe de Información en Ciencias de la Salud que daría origen a las Bibliotecas Virtuales en Salud conocidas como BVS, que luego tendrían derivaciones soportadas por el consorcio Biblioteca Regional de Medicina, la Organización Panamericana de la Salud y la Organización Mundial de la Salud (BIREME/ OPS/OMS).

Fue claro desde el comienzo que América Latina consideró de importancia crítica que un área como la salud tuviera conocimiento de la epidemiologia y que los desarrollos en biomedicina estuvieran disponibles en forma libre. Posteriormente aparecieron las iniciativas de Scielo y Redalyc, la primera originada en Brasil y la segunda en México. Scielo con un énfasis en biomedicina y Redalyc en ciencias sociales, y finalmente, desde España, la iniciativa Dialnet. Estos proyectos se expandieron en forma distinta por América latina, Scielo busco descentralizar la operación, delegando así a cada país la generación de la infraestructura necesarias para su funcionamiento, mientras que Redalyc creció con un modelo concentrado en un solo país y en una sola institución. Ambos sistemas 
terminaron multiplicando la visibilidad de una parte del conocimiento producido por y para la región. No obstante, Scielo concentrado en ampliar la visibilidad internacional y creyendo firmemente en las métricas derivadas de WoS entrego una parte de sus plataformas a la empresa Clarivate, esta operación no ha sido clara para todos los actores y ha generado muchas preguntas sobre la transparencia de su gobernanza. Redalyc, por su parte, ha buscado alejarse de las métricas tradicionales, y además de criticarlas, ha tratado de generar otras métricas; su principal debilidad ha sido concentrarse en un solo actor: la Universidad Autónoma del Estado de México (UAEM), que financia toda la infraestructura y operación. Esta situación hace a Redalyc vulnerable a las desafortunadas crisis económicas y políticas de las instituciones, y la ignorancia general respecto al valor irremplazable de una iniciativa como esta para la región no los ayuda. Como he dicho en múltiples contextos, Redalyc es un patrimonio cultural de México y de Latinoamérica, que ha confiado sus contenidos a la plataforma, una Biblioteca de Alejandría Latinoamericana digital. Redalyc ha contribuido en forma única a visibilizar los contenidos producidos por y para la región, con un énfasis especial en los resultados de investigación que representan el ADN cultural de Latinoamérica: sus revistas de ciencias sociales y humanas.

A pesar de lo anterior, la postura de Redalyc en el último tiempo ha estado orientada a la idea de que no pueden ser admisibles los modelos que aceptan acuerdos transformacionales tales como los que sugiere el Plan $\mathrm{S} y$, por tanto, no puede admitirse ningún pago en el circuito de comunicación, ninguna parte de las publicaciones académicas puede ser comercial. Sin embargo es importante tener en cuenta que Latinoamérica no es uniforme en su ecosistema de producción y formación de conocimiento. Como lo indicó el más reciente diagnóstico de la educación superior en Iberoamérica que publicó la OEI (2019), un número creciente de países de la región tienen educación universitaria privada y no pública. Esta desafortunada realidad tiene como consecuencia que los procesos de publicación en la región tengan costos que no son siempre transferibles a los que pagan por la educación, y tristemente se opta por no producir revistas o por eliminar las que se tienen, así como por incentivar el envío de los contenidos a revistas con muros de pago o con pagos transferidos a los investigadores (APC). Además, es claro que los países con ecosistemas diversos y con menos recursos son altamente vulnerables a múltiples intereses y presiones, como se ha mencionado anteriormente (LópezLópez, 2019a).

Por otro lado, los investigadores también se encuentran atrapados en esta compleja dinámica ya que deben tener en cuenta que la publicación de artículos que es (en parte) financiada por los fundadores del Plan S demandará que estas publicaciones sean de acceso abierto. En el caso de América Latina esto aumentará la dificultad puesto que los sistemas de incentivos están atados a las métricas ya criticadas que, en general, están asociadas a las revistas de las grandes empresas editoriales que cuentan con muros de pago o con APC que simplemente no podrán ser cubiertos por los países de la región con economías frágiles. Por tanto, es responsabilidad de la gobernanza científica de la región, los editores y los investigadores, el prepararse para estas nuevas condiciones.

En este sentido, Latinoamérica debería incentivar el publicar en revistas de calidad en acceso abierto. Al mismo tiempo, el Plan S puede financiar a los investigadores y presionar a las revistas con APC a regular sus costos, a hacerlos transparentes y a alinear los pagos de APC con la capacidad de pago de los países (la paridad del poder de compra Purchasing Power Parity (PPP)). Es evidente que lo ideal es el modelo Diamante del acceso abierto, pero los modelos Dorados, que requiere un pago de APC de parte de los autores o sus instituciones/financiadores, pueden hacerse más justos y transparentes, y además permitirán publicar. También está el modelo verde, en el que los autores pueden subir sus artículos y compartirlos en post-print sin la necesidad de pagar el APC, en el caso de los que son cubiertos por la subscripciones. Estos modelos de negocio son parte de nuestro ecosistema de comunicación científica y no se 
unificaran en el corto plazo, es urgente encontrar soluciones que lleven a modelos de gestión de comunicación científica sostenibles y razonables para los diferentes actores del sistema.

Evidentemente, el Plan $S$ tiene un papel central para la ciencia abierta en el mundo por cuanto está buscando desarrollar múltiples soluciones en un contexto en el que es claro que las grandes casas editoriales no cederán sus intereses del multimillonario negocio. En ese sentido, la Coalicion S necesita seguir sumando integrantes: hoy cuenta con 24 miembros, incluyendo la Organización Mundial de la Salud, La Comisión Europea, el Consejo Europeo de Investigación, la United Kingdom Research and Innovation (UKRI), la Fundación Bill y Melinda Gates, el Programa para la investigación y entrenamiento en enfermedades tropicales (TDR), programa al que están asociados la UNICEF y el Banco Mundial, y el Programa de las Naciones Unidas para el Desarrollo (UNDP), entre otras. Hay un sesgo notable hacía las entidades ligadas a la salud global que es explicado por las amenazas mundiales a la salud que existen, por lo que se hace aún más urgente encontrar fórmulas viables para desarrollar el acceso abierto, espero que mi nuevo rol como embajador del Plan S me permita contribuir en esta dirección.

\section{Referencias}

cOAlition S. (2019). Accelerating the transition to full and immediate Open Access to scientific publications. Bruselas, Bélgica: Science Europe https://www.coalition-s.org/wp-con tent/uploads/PlanS_Principles_and_Imple mentation_310519.pdf

DORA (2012). San Francisco Declaration on Research Assessment. https://sfdora.org/re ad/es/

Flórez-Carranza, F. (2018). Nociones de calidad e impacto: el lugar de las revistas indexadas de ciencias jurídicas en el nuevo sistema colombiano de competitividad, ciencia, tecnología e innovación. Vniversitas,
67(137). https://doi.org/10.11144/Javerian a.vj137.ncei

López-López, W. (2019a). El sistema colombiano de medición de revistas Publindex: Las paradojas de un sistema que devalúa el conocimiento producido localmente. ASEUC. Recuperado de http://amelica.org/index.php/index-entr adas/

López-López, W. (2019b). Ecosistema del conocimiento en América Latina: el acceso abierto, métricas, paradojas y contradicciones. Universitas Psychologica, 18(4), 1-2. http://doi.org/10.11144/Javeria na.upsy18-4.ecal

OEI. (2019). Informe de diagnóstico de la educación superior en Iberoamérica. https://www.oei.es/uploads/fil es/news/Science-Science-and-University/1 603/informediagnostico2019.pdf

Schiltz, M. (2018) Science without publication paywalls: cOAlition $S$ for the Realization of Full and Immediate Open Access. PLoS Med 15(9), 1-4. https://doi.org/10.1371/jou rnal.pmed.1002663

UNESCO. (2011). A Global Perspective on Open Access. UNESCO Netherlands National Commission for UNESCO. Recuperado de http://www.unesco.org/new/fileadmin/ MULTIMEDIA/HQ/CI/CI/pdf/themes/acc ess to knowledge societies/open access/e n\%20-\%20UNESCO\%20 expert\%20meeti ng\%200pen\%20Access\%20conclusions.p df 